2. Ramos L, Isenberg D. Rituximab: the Lupus Journey. Current Treatment Options in Rheumatology 2015;1(1):30-41.

3. Murphy G, Isenberg DA. New therapies for systemic lupus erythematosus - past imperfect, future tense. Nature Reviews Rheumatology 2019;15(7):403-12.

4. Hennessey A, Lukawska J, Cambridge G, et al. AB0443 Infusion reactions to rituximab in systemic lupus erythematosus: a retrospective analysis. Annals of the Rheumatic Diseases 2017;76(Suppl 2):1205-05.

5. Alexander T, Cheng Q, Klotsche J, et al. Proteasome inhibition with bortezomib induces a therapeutically relevant depletion of plasma cells in SLE but does not target their precursors. European Journal of Immunology 2018;48(9):1573-79.

\section{TARGETING INTERLEUKIN 12/23}

Ronald van Vollenhoven. Amsterdam University Medical Centers, and Amsterdam Rheumatology \& Immunology Center

\subsection{6/lupus-2019-la.15}

Multiple novel approaches to treating systemic lupus erythematosus (SLE) in recent years have resulted in the approval of a single B-cell directed therapy, but also in the failure of several promising drug candidates. As many immunological pathways are disrupted in SLE, ${ }^{1}$ it was recognized that immunomodulatory drugs approved for other conditions might also be effective in SLE. Grammer et al. employed a systematic analysis of existing drugs and found that the interleukin (IL)12/23 antagonist ustekinumab had a relatively high a priori likelihood of being effective in SLE. ${ }^{2}$ Thus, IL12 plays an essential role in the activation and function of various $\mathrm{T}$ cell subsets seen in the inflammatory infiltrates in the tissues of patients with SLE, including follicular T-helper cells, T-helper1 cells, and cytotoxic T cells; while IL-23 drives the expansion and survival of pathogenic T-helper-17 cells and decreases IL2 production thereby diminishing regulatory $\mathrm{T}$ cell activity. ${ }^{3}$ Moreover, in animal models of SLE, the selective deletion of the p40 subunit, which is shared by IL12 and IL23, resulted in decreased disease activity; ${ }^{4}$ and several SLE-risk genes are related to the IL12 pathway. The IL12/23 antagonist monoclonal antibody ustekinumab binds the p40 subunit and thereby interferes with the activity of both IL12 and IL23. It has been approved in many countries for the treatment of psoriatic arthritis, psoriasis and Crohn's disease, and there is extensive clinical experience with the drug in patients with these diseases where the safety profile is considered favorable.

Based on these considerations, a Phase II clinical trial of ustekinumab was conducted in patients with active SLE despite conventional background therapy. The patient population in this trial was reflective of that seen in practice and in most clinical trials, with a large predominance of women and the most commonly affected organ systems being the skin and the joints. In the 24 week randomized, controlled portion of the trial, a statistically significant difference was seen in the response rate of patients on ustekinumab versus placebo. Thus, in the ustekinumab group $62 \%$ of patients achieved the SRI-4 versus $33 \%$ in the placebo group $(p=0.0057) .^{5}$ Differences favoring ustekinumab were also demonstrated for some other outcomes such as the individual measures for skin and joint involvement and the number of flares. After Week 24, all patients continued on active ustekinumab treatment. At Week 48, the original ustekinumab group had maintained the responses, while the original placebo group showed improved outcomes. The safety and tolerability of ustekinumab in this relatively small trial were consistent with the much larger experience in other diseases and generally good. A Phase III clinical trial to confirm and extend these results is currently underway (NCT03517722).

\section{Learning objectives}

- Explain the significance of the IL12/23 pathway in the pathogenesis of SLE

- Describe the existing evidence for ustekinumab treatment in SLE

- Discuss the potential for IL12/23 blockade in the future treatment of SLE

\section{REFERENCES}

1. Kaul A, Gordon C, Crow MK, et al. Systemic lupus erythematosus. Nature Reviews Disease Primers 2016;2(1).

2. Grammer AC, Ryals MM, Heuer SE, et al. Drug repositioning in SLE: crowd-sourcing, literature-mining and Big Data analysis. Lupus 2016;25(10):1150-70.

3. Dai H, He F, Tsokos GC, et al. IL-23 Limits the Production of IL-2 and Promotes Autoimmunity in Lupus. The Journal of Immunology 2017;199(3):903-10.

4. Kikawada E, Lenda DM, Kelley VR. IL-12 Deficiency in MRL-Faslpr Mice Delays Nephritis and Intrarenal IFN- $\gamma$ Expression, and Diminishes Systemic Pathology. The Journal of Immunology 2003;170(7):3915-25.

5. van Vollenhoven RF, Hahn BH, Tsokos GC, et al. Efficacy and safety of ustekinumab, an IL-12 and IL-23 inhibitor, in patients with active systemic lupus erythematosus: results of a multicentre, double-blind, phase 2, randomised, controlled study. The Lancet 2018;392(10155):1330-39.

\section{TARGETING NOVEL INTRACELLULAR PATHWAYS}

Thomas Dörner. Charite University Hospitals Berlin, Germany

\subsection{6/lupus-2019-la.16}

Systemic lupus erythematosus SLE is characterised by abnormalities in cellular and humoral immunity, while disturbances in cytokine production became very clear in recent years. Identification of increased IL-6, IL-17, IL-12 and IL-23, BAFF, and especially type I IFN production by different cell types, provided the rationale for targeting these cytokines and their corresponding cytokine receptors using biologics. Since these cytokine activate various intracellular pathways, such as Jak/Stat signaling, activation of the $\mathrm{Nf \kappa B}$ or using spleen tyrosine kinase (Syk), Bruton's tyrosine kinase (BTK), small molecules inhibiting these pathways are being investigated in various clinical studies. It should be emphasised that most of the above mentioned intracellular pathways may vary between different immune cells and tissues and can have interactions which have not been fully delineated. However, certain strategies target multiple key pathways along with inhibiting various cytokines (multiple targeting therapy $)^{1}$ which holds the promise to cover broadly heterogeneous SLE, a therapeutic principle that has already been introduced in antihypertensive and ant infectious treatment algorithms.

As a first example in patients with SLE, treatment with the Jak1/Jak2 blocking agent (jakinib) baricitinib showed improvements of skin and joint manifestations among patients with a daily dose of $4 \mathrm{mg} / \mathrm{d}$ but less pronounced under $2 \mathrm{mg} / \mathrm{d}$ in a Phase II trial over 24 weeks. $^{2}$ Another Phase Ib/IIa trial using tofacitinib as Jak1/Jak3 selective inhibitor in SLE has been reported without substantial safety concerns and early signs of efficacy. ${ }^{3}$ In addition to jakinib in studies with SLE, there are also trials of inhibitors of other pathways (BTK, Syk etc.) that hold promise for a new era of more efficacious and well tolerated therapies that may address the current and substantial need for the effective treatment of SLE. 